\title{
BIBLIOGRAPHY
}

1. P. Erdös and A. H. Stone, Some remarks on almost periodic transformations, Bull. Amer. Math. Soc. vol. 51 (1945) pp. 126-130.

2. W. H. Gottschalk, Powers of homeomorphisms with almost periodic properties, Bull. Amer. Math. Soc. vol. 50 (1944) pp. 222-227.

University of Pennsylvania and UNIVERSITY OF VIRGINIA

\section{A REMARK ON DENSITY CHARACTERS}

\section{EDWIN HEWITT ${ }^{1}$}

Let $X$ be an arbitrary topological space satisfying the $T_{0}$-separation axiom [1, Chap. $1, \S 4$, p. 58]. ${ }^{2}$ We recall the following definition [3, p. 329].

Definition 1. The least cardinal number of a dense subset of the space $X$ is said to be the density character of $X$. It is denoted by the symbol $\Xi(X)$.

We denote the cardinal number of a set $A$ by $|A|$.

Pospísil has pointed out [4] that if $X$ is a Hausdorff space, then

$$
|X| \leqq 2^{2 \Xi(X)} \text {. }
$$

This inequality is easily established. Let $D$ be a dense subset of the Hausdorff space $X$ such that $|D|=\Xi(X)$. For an arbitrary point $p \in X$ and an arbitrary complete neighborhood system $v_{p}$ at $p$, let $\mathcal{D}_{p}$ be the family of all sets $U \cap D$, where $U \in V_{p}$. Thus to every point of $X$, a certain family of subsets of $D$ is assigned. Since $X$ is a Hausdorff space, $\mathcal{D}_{p} \neq \mathcal{D}_{q}$ whenever $p \neq q$, and the correspondence assigning each point $p$ to the family $\mathcal{D}_{p}$ is one-to-one. Since $X$ is in one-to-one correspondence with a sub-hierarchy of the hierarchy of all families of subsets of $D$, the inequality (1) follows.

It may be remarked in passing that the inequality (1) does not obtain for all $T_{1}$-spaces. Let $\mathfrak{m}$ be a cardinal number greater than $2^{c}$, where $\mathfrak{c}=2 \aleph_{0}$. Let $Z$ be a $T_{1}$-space of cardinal number $\mathfrak{m}$ and with the property that the only closed proper subsets of $Z$ are finite or

Received by the editors March 5, 1946.

${ }^{1}$ Post-service fellow of the John Simon Guggenheim Memorial Foundation.

2 Numbers in brackets refer to the Bibliography at the end of the paper. 
void. Then it is obvious that $\Xi(Z)=\aleph_{0}$, and that (1) does not obtain for the space $Z$.

For some Hausdorff spaces, the equality

$$
|X|=2^{2^{\Xi(X)}}
$$

obtains. Pospísiil [4] has constructed a large family of such Hausdorff spaces, and has shown [5] that the Stone-Cech $\beta$ for any discrete infinite space satisfies it as well. It is the purpose of this note to exhibit another class of Hausdorff spaces for which (2) holds.

TheOREM. Let $\Lambda$ be an index class such that $|\Lambda|=2^{\mathfrak{m}}$ where $\mathfrak{m}$ is an infinite cardinal number. Let $\left\{X_{\lambda}\right\}_{\lambda \in \Lambda}$ be a family of Hausdorff spaces such that $\left|X_{\lambda}\right| \geqq 2$ and $\Xi\left(X_{\lambda}\right) \leqq \mathfrak{m}$ for all $\lambda \in \Lambda$. Then $\left|\mathfrak{P}_{\lambda \in \Lambda} X_{\lambda}\right|=2^{2^{\mathfrak{m}}}$ and $\Xi\left(\mathfrak{P}_{\lambda \in \Lambda} X_{\lambda}\right)=\mathfrak{m}$.

Proof. We first consider the set $\Lambda$ as a topological space itself. Clearly, it may be put into one-to-one correspondence with the Cartesian product $\mathfrak{P}_{\mu \in M} A_{\mu}$, where each $A_{\mu}$ is a Hausdorff space containing exactly two points and the index class $M$ has cardinal number $\mathfrak{m}$. As is well known, this Cartesian product is a bicompact Hausdorff space with cardinal number $2^{\mathfrak{m}}$ and a basis of open sets with cardinal number $\mathfrak{m}$. We may consequently regard $\Lambda$ as being a Hausdorff space with a basis $\mathcal{B}$ of open sets such that $|\mathcal{B}|=\mathfrak{m}$.

Let $y^{0}=\left\{q_{\lambda}{ }^{0}\right\}$ be a fixed point in the space $P_{\lambda \in \Lambda} X_{\lambda}$. Let $D_{\lambda}$ be a dense subset in $X_{\lambda}$ such that $\left|D_{\lambda}\right|=\Xi\left(X_{\lambda}\right) \leqq \mathfrak{m}$. If $\alpha_{0}$ is the least ordinal number with corresponding cardinal number $\mathfrak{m}$, then each set $D_{\lambda}$ can be so well ordered that

$$
D_{\lambda}=\left\{p_{\lambda}^{1}, p_{\lambda}^{2}, p_{\lambda}^{3}, \cdots, p_{\lambda}^{\alpha}, \cdots\right\},
$$

$\alpha<\alpha_{0}$.

If $\left|D_{\lambda}\right|<\mathfrak{m}$, then the elements $p_{\lambda}{ }^{\alpha}$ may be all taken identical from a certain point on. Of course, if $\left|D_{\lambda}\right|=\mathfrak{m}$, no repetitions need occur.

Let $\left\{\Lambda_{1}, \cdots, \Lambda_{n}\right\}$ be an arbitrary family of disjoint sets in $\mathcal{B}$, and let $\left\{\alpha_{1}, \cdots, \alpha_{n}\right\}$ be arbitrary ordinal numbers all less than $\alpha_{0}$. Let $x\left(\Lambda_{1}, \cdots, \Lambda_{n} ; \alpha_{1}, \cdots, \alpha_{n}\right)=\left\{r_{\lambda}\right\}$ be the point in $\mathfrak{B}_{\lambda \in \Lambda} X_{\lambda}$ such that $r_{\lambda}=p_{\lambda}{ }^{\alpha_{i}}$ for all $\lambda \in \Lambda_{i}, i=1,2,3, \cdots, n$, and $r_{\lambda}=q_{\lambda}{ }^{0}$ for $\lambda \in \Lambda \cap\left(\sum_{i=1}^{n} \Lambda_{i}\right)^{\prime}$. Let $W$ be the set of all points $x\left(\Lambda_{1}, \cdots, \Lambda_{n}\right.$; $\left.\alpha_{1}, \cdots, \alpha_{n}\right)$ as $\left\{\Lambda_{1}, \cdots, \Lambda_{n}\right\}$ and $\left\{\alpha_{1}, \cdots, \alpha_{n}\right\}$ assume all possible values. It is clear that $|W|=\sum_{n=1}^{\infty} \mathfrak{m}^{n} \cdot \mathfrak{m}^{n}=\boldsymbol{N}_{0} \cdot \mathfrak{m}=\mathfrak{m}$. Furthermore, $W$ is dense in $\mathfrak{B}_{\lambda \in \Lambda} X_{\lambda}$. Let $G$ be an arbitrary non-void open set in $\mathfrak{P}_{\lambda \in \Lambda} X_{\lambda}$. By the definition of open sets and neighborhoods in a Cartesian product (see, for example, [2, pp. 829-830]), there exist a finite subset $\left\{\lambda_{1}, \cdots, \lambda_{m}\right\}$ of $\Lambda$ and sets $U_{\lambda_{1}} \cdots, U_{\lambda_{m}}$, where $U_{\lambda_{i}}$ is an open set in $X_{\lambda_{i}}$, with the property that $G$ contains all points $\left\{s_{\lambda}\right\}$ 
of $\mathfrak{F}_{\lambda \in \Lambda} X_{\lambda}$ such that $s_{\lambda_{i}} \in U_{\lambda_{i}}$ for $i=1,2,3, \cdots, m$. The sets $D_{\lambda}$ being dense in the spaces $X_{\lambda}$, there is a point $p_{\lambda_{i}} \alpha_{i} \in D_{\lambda_{i}}$ such that $p_{\lambda_{i}}{ }^{\alpha_{i}} \in U_{\lambda_{i}}$ $(i=1,2,3, \cdots, m)$. Since $\Lambda$ is a Hausdorff space under the topology defined by $\mathcal{B}$, there are sets $\Lambda_{1}, \cdots, \Lambda_{m}$ in $\mathcal{B}$ such that $\Lambda_{i} \cap \Lambda_{j}=0$ for $i \neq j$ and such that $\lambda_{i} \in \Lambda_{i}$ for all $i=1,2,3, \cdots, m$. It is obvious that the point $x\left(\Lambda_{1}, \cdots, \Lambda_{m} ; \alpha_{1}, \cdots, \alpha_{m}\right)$ is in the set $W \cap G$. Having a nonvoid intersection with an arbitrary nonvoid open set in $\mathfrak{P}_{\lambda \in \Lambda} X_{\lambda}, W$ is dense in $\mathfrak{P}_{\lambda \in \Lambda} X_{\lambda}$.

It follows from the definition of $\Xi\left(\mathfrak{P}_{\lambda \in \Lambda} X_{\lambda}\right)$ and the equality $|W|=\mathfrak{m}$ that $\mathbb{\Xi}\left(\mathfrak{P}_{\lambda \in \Delta} X_{\lambda}\right) \leqq \mathfrak{m}$. On the other hand, we have

$$
\left|\mathfrak{P}_{\lambda \in \Delta} X_{\lambda}\right| \geqq \mathfrak{m}^{|\Delta|}=2^{2^{\mathfrak{m}}} \text {. }
$$

Hence, by virtue of the inequality (1), it follows that

$$
\left|\mathfrak{P}_{\lambda \in \Lambda} X_{\lambda}\right|=2^{2^{\mathfrak{m}}}
$$

and

$$
\Xi\left(\mathfrak{P}_{\lambda \in \Lambda} X_{\lambda}\right)=\mathfrak{m} .
$$

This completes the proof.

For a result similar to this, see [6].

The foregoing theorem, applied to various well known spaces, yields curious results.

COROLLARY 1. The space of all real-valued functions of a real variable with the Cartesian product topology contains a countable dense subset.

COROLLARY 2. The space of all characteristic functions defined on a set of cardinal number $2 \boldsymbol{s}_{0}$ contains a countable dense subset under the Cartesian product topology.

\section{BIBLIOGRAPHY}

1. P. Alexandroff and H. Hopf, Topologie, vol. 1, Springer, Berlin, 1935.

2. Eduard Cech, On bicompact spaces, Ann. of Math. vol. 38 (1937) pp. 823-844.

3. Edwin Hewitt, A problem of set-theoretic topology, Duke Math. J. vol. 10 (1943) pp. 309-333.

4. Bedřich Pospišil, Sur la puissance d'un espace contenant une partie dense de puissance donnée, Casopis pro pěstování Matematiky a Fysiky vol. 67 (1937-1938) pp. 89-96.

5. - Remark on bicompact spaces, Ann. of Math. vol. 38 (1937) pp. 845-846.

6. E. S. Pondiczery, Power problems in abstract spaces, Duke Math. J. vol. 11 (1944) pp. 835-837.

The Institute for Advanced Study and Princeton University 